Marquette University

e-Publications@Marquette

Biomedical Engineering Faculty Research and

Publications

Biomedical Engineering, Department of

$7-1-2012$

\title{
Adaptive Optics Scanning Ophthalmoscopy with Annular Pupils
}

Yusufu N. Sulai

University of Rochester

Alfredo Dubra

Marquette University

Accepted version. Biomedical Optics Express, Vol. 3, No. 7 (July 2012): 1648-1661. DOI. (C) 2012 Optical Society of America.

This is an open-access article distributed under the terms of the Creative Commons AttributionNoncommercial-No Derivative Works 3.0 Unported License, which permits download and redistribution, provided that the original work is properly cited. This license restricts the article from being modified or used commercially. 


\title{
Adaptive optics scanning ophthalmoscopy with annular pupils
}

\author{
$\underline{\text { Yusufu N. Sulai }}^{1}$ and Alfredo Dubra ${ }^{2,3,4, *}$ \\ ${ }^{1}$ The Institute of Optics, University of Rochester, Rochester, NY 14627, USA \\ ${ }^{2}$ Department of Ophthalmology, Medical College of Wisconsin, Milwaukee, WI 53226, USA \\ ${ }^{3}$ Department of Biophysics, Medical College of Wisconsin, Milwaukee, WI 53226, USA \\ ${ }^{4}$ Department of Biomedical Engineering, Marquette University, Milwaukee, WI 53233, USA \\ *Email: adubra@mcw.edu
}

Received 2012 Mar 13; Revised 2012 Apr 25; Accepted 2012 Jun 1.

Copyright $@ 2012$ Optical Society of America

This is an open-access article distributed under the terms of the Creative Commons Attribution-Noncommercial-No Derivative Works 3.0 Unported License, which permits download and redistribution, provided that the original work is properly cited. This license restricts the article from being modified or used commercially.

This article has been cited by other articles in PMC.

Annular apodization of the illumination and/or imaging pupils of an adaptive optics scanning light ophthalmoscope (AOSLO) for improving transverse resolution was evaluated using three different normalized inner radii $(0.26,0.39$ and 0.52$)$. In vivo imaging of the human photoreceptor mosaic at 0.5 and $10^{\circ}$ from fixation indicates that the use of an annular illumination pupil and a circular imaging pupil provides the most benefit of all configurations when using a one Airy disk diameter pinhole, in agreement with the paraxial confocal microscopy theory. Annular illumination pupils with 0.26 and 0.39 normalized inner radii performed best in terms of the narrowing of the autocorrelation central lobe (between 7 and 12\%), and the increase in manual and automated photoreceptor counts ( 8 to $20 \%$ more cones and 11 to $29 \%$ more rods). It was observed that the use of annular pupils with large inner radii can result in multi-modal cone photoreceptor intensity profiles. The effect of the annular masks on the average photoreceptor intensity is consistent with the Stiles-Crawford effect (SCE). This indicates that combinations of images of the same photoreceptors with different apodization configurations and/or annular masks can be used to distinguish cones from rods, even when the former have complex multi-modal intensity profiles. In addition to narrowing the point spread function transversally, the use of annular apodizing masks also elongates it axially, a fact that can be used for extending the depth of focus of techniques such as adaptive optics optical coherence tomography (AOOCT). Finally, the positive results from this work suggest that annular pupil apodization could be used in refractive or catadioptric adaptive optics ophthalmoscopes to mitigate undesired back-reflections.

OCIS codes: (110.1080) Active or adaptive optics, (220.1230) Apodization, (170.3880) Medical and biological imaging, (170.4460) Ophthalmic optics and devices

\section{Introduction}

Current clinical ophthalmoscopes form images of the retina by delivering and collecting light through non overlapping portions of the eye's pupil in order to avoid undesired reflections from the cornea and crystalline lens. Typically, the outermost part of the pupil is used for illumination and the central 2-3 mm are used for either observation (e.g. direct ophthalmoscope) or imaging (e.g. fundus cameras). When such a small area of the pupil is used for light collection, the image resolution is limited by diffraction when good spectacle correction has been achieved. For larger pupils, however, retinal images are blurred by the monochromatic aberrations of the optics of the eye. Ophthalmic instruments that incorporate adaptive optics (AO) [1- 15$]$ can compensate these aberrations, potentially reaching the classical Rayleigh resolution limit using larger diameter pupils. AO wavefront correction over a large pupil has resulted in an increase in transverse and axial resolution that has allowed in vivo visualization of multiple microscopic retinal structures in human and animal models, including but not limited to: the cone photoreceptor mosaic $[\underline{1}, \underline{6}, \underline{16}]$, the retinal pigment epithelial cell mosaic $[\underline{8}, \underline{17}, \underline{18}]$, leukocytes [19], the retinal capillary vasculature [19-21], fluorescently labeled retinal ganglion cells [ [ ] ], the lamina cribrosa [22-24], and more recently, the rod photoreceptor mosaic [25, 26]. Unfortunately, these structures cannot be resolved in all subjects due to a number of factors, one of them being the reduction in pupil diameter that comes with aging [27] which results in a proportional reduction in transverse resolution and a quadratic reduction in signal. This 
issue alone severely limits the potential applicability of $\mathrm{AO}$ for clinical diagnosis, given that aging is a major risk factor in the most prevalent blinding conditions, including age-related macular degeneration, glaucoma and diabetic retinopathy [28-30]. It is therefore essential to explore methods for further improving on the transverse resolution provided by AO.

Here, the use of pupil apodization, namely the manipulation of the amplitude and/or phase of the light at the pupil planes is explored as a means to narrow the point spread function (PSF) of the optical system. In particular, we evaluate the use of circular opaque masks centered on the pupils of the illumination and/or imaging paths $[\underline{9}, \underline{31}, \underline{32}]$ of a reflectance AOSLO, with the goal of improving its transverse resolution. The resolution improvement provided by these apodization masks measured in terms of the Rayleigh criterion [33], stems from the attenuation of the lower spatial frequency content of the images. Unlike other superresolving techniques, such as structured illumination [34], pupil apodization does not increase the spatial frequency bandwidth of the image, and can in fact, be thought of as equivalent to edge-enhancing methods used in image processing.

The amplitude $h_{1}$ of the PSF of a diffraction-limited rotationally symmetric lens with unit radius and a central obscuration of radius $\varepsilon$ under the paraxial approximation is given by [풀

$$
h_{1}(v, u) \propto \int_{\varepsilon}^{1} J_{0}(v \rho) e^{-i u \rho^{2} / 2} \rho \mathrm{d} \rho
$$

where $v$ and $u$ are dimensionless radial and axial coordinates, defined as

$$
v=\frac{2 \pi}{\lambda}\left(\frac{a}{f}\right) \sqrt{x^{2}+y^{2}}, \quad u=\frac{2 \pi}{\lambda}\left(\frac{a}{f}\right)^{2} z
$$

Here, $\lambda$ denotes light wavelength, $f$ is the lens effective focal length, $a$ is the lens radius (in this case also the mask outer radius), and $x, y$ and $z$ are the Cartesian coordinates with the $z$-axis along the lens axis of symmetry, which is also the direction of propagation of light. The combination of a rotationally symmetric lens with the type of mask described here is usually referred to as an annular lens. Figure 1 shows the normalized intensity of the PSF of such diffraction-limited annular lenses for the different values of $\varepsilon$ that will be evaluated experimentally. A small transverse narrowing and axial elongation of the PSF central lobe can be observed with increasing $\varepsilon$. This means that any improvement in transverse resolution will come at the price of signal and axial resolution degradation. In confocal scanning instruments, the latter translates into a loss of axial sectioning, or equivalently, an increase in depth of focus. It should also be noted that this type of apodization increases the relative intensity of the PSF side lobes in the geometrical image plane. This should be kept in mind when imaging small dim objects in close proximity to brighter objects.

Fig. 1

Binary annular pupil transmission masks with various relative inner radii $\varepsilon$ (top) and the corresponding PSFs when used in diffraction-limited circularly symmetric lenses (bottom). The microscopy dimensionless coordinates $u$ and $v$, defined in Eq. ...

A diffraction-limited confocal AOSLO is effectively a confocal scanning microscope, and as such, its PSF can be calculated by combining the effects of the imaging and illumination PSFs, as well as the finite size of the light detector. In an AOSLO, the effective size of the light detector is determined by the confocal aperture that precedes the light detector, while in AOOCT it is determined by the core of the optical fiber that is typically used to collect the light to be directed to the light detector. The intensity of the confocal microscope PSF is calculated as

$$
I \propto\left|h_{\text {illumination }}\right|^{2}\left(\left|h_{\text {imaging }}\right|^{2} \otimes p\right)
$$


where $p$ is a 2-dimensional function describing the detector size and $\otimes$ denotes convolution [36]. By combining Eqs. (1) and (3) we calculated the PSF for different values of $\varepsilon$ and detector size, as well as all four combinations of annular and circular lenses in the illumination and imaging pupils, and then determined the transverse and axial resolution in terms of the Rayleigh criterion [33] The resulting resolution plots, shown in Fig. 2 , do not take into account the wave-guiding properties of the photoreceptors that are used to explain the SCE, and therefore, departures from these theoretical predictions in our experimental results should be expected.

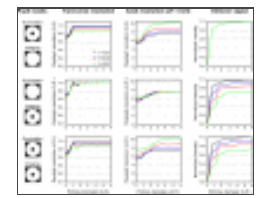

Fig. 2

Transverse resolution (left), axial resolution (center) and energy detected (right) in a confocal scanning instrument, as a function of the detector size for various relative pupil obscuration radii $(\varepsilon)$, in terms of Airy disk radii (A.R.). The ...

\subsection{Transverse resolution}

The most distinctive feature in all the plots in Fig. 2 is the transition that occurs when the detector size is comparable to that of the Airy disk. The resolution plots on the left remind us that in order to get the maximum improvement in transverse resolution provided by the use of a confocal aperture (up to 20\%), its size has to be no larger than half the Airy disk. For AO ophthalmoscopes though, this benefit will only materialize if the AO compensation of the monochromatic aberrations is such that close to diffraction-limited performance is achieved. The transverse resolution benefit of using a sub-Airy disk confocal aperture comes at the price of reduced light collection efficiency, as indicated by the plots on the right column, which could be a major limitation when the number of photons that can be delivered to the sample is limited. This is usually the case in fluorescence microscopy and in ophthalmoscopy where bleaching and thermal or photochemical damage to the retina, are a concern.

The comparison of the transverse resolution curves for apodizing masks with different $\varepsilon$ shows that the apodization of the illumination path improves resolution for all detector sizes, with the largest benefit taking place for the larger confocal aperture. Interestingly, apodizing the pupil in the imaging path provides no benefit other than for confocal apertures much smaller than the Airy disk. The asymmetry between apodizing the illumination or the imaging pupils stems from the convolution of the imaging PSF and the detector function, shown in Eq. (3). The convolution effectively blurs any narrowing of the PSF of the apodized imaging optics other than for detectors smaller than a quarter of an Airy disk, for which the resolution improvement is approximately 5\%. But in obtaining this small benefit the detected signal is reduced by almost an order of magnitude, which could lead to an unacceptably low signal-to-noise ratio (SNR). The plots that correspond to the simultaneous apodization of both the illumination and imaging pupils are similar to those that result from apodizing the illumination pupil only, with the added disadvantage of the detected signal reduction that comes with the annular imaging pupil. The sole situation in which the apodization of both pupils shows an improvement over the apodization of the illumination pupil alone ( $3 \%$ for $\varepsilon=0.52)$, is for confocal apertures smaller than one quarter of the Airy disk.

\subsection{Axial resolution}

The main advantage of using confocal scanning instruments for retinal imaging comes from the axial sectioning that fundus cameras cannot provide. The finite size of the confocal aperture in the image plane means that some out-of-focus light is not detected, thus enhancing the contrast of the in-focus feature. The plots in the middle column of Fig. 2 show that axial resolution for all values of $\varepsilon$ and pupil apodization configurations is better for detectors comparable to or smaller than the Airy disk. Unlike the transverse case, there is no axial resolution benefit in using confocal apertures much smaller than the Airy disk.

Consistent with the PSF axial widening due to increasing $\varepsilon$ in annular lenses (shown in Fig. 1), the apodization of the illumination pupil in a confocal scanning instrument results in degradation the of axial resolution. This increase of the depth of focus could be beneficial for applications such as AOOCT. Apodization of the imaging path, however, does not affect the axial sectioning, other than for detector sizes smaller than the Airy disk. Finally, when both pupils are apodized, the degradation in axial resolution is approximately the sum total of the resolution loss that results from apodizing each pupil separately.

\subsection{Modulation transfer function}

The origin of the changes in transverse resolution with the inner radius and configuration of the pupil apodization mask can be interpreted in terms of its effect on the spatial frequency content of the image, as illustrated by the plots in Fig. 3 . Typically, MTF curves are normalized to unit amplitude at zero frequency. In the plots presented here, however, the amplitude at zero frequency is proportional to the detected power, assuming that the same number of photons per second is delivered to the sample. In this way, we facilitate the comparison of MTF amplitude for different apodization configurations at any given frequency, given the same light exposure. The curves were generated assuming that $680 \mathrm{~nm}$ light was used to image an eye with a $17 \mathrm{~mm}$ focal length 


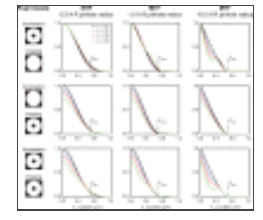

Fig. 3

MTF of a confocal microscope with different apodizing binary mask combinations (one per row) with different radii (indicated by color) and pinhole sizes (one per column). These plots were calculated assuming $680 \mathrm{~nm}$ light, an eye with $17 \mathrm{~mm}$ focal length ...

The plots show that for confocal pinholes smaller than the Airy disk the MTF extends beyond the cut off spatial frequency $\left(f_{\text {max }}\right)$ of a non-scanning incoherent imaging system that uses uniform illumination at the object plane (e.g. AO fundus camera). This increased bandwidth is the reason for the superior lateral resolution of confocal microscopes over bright field microscopes. When using detectors equal to or larger than the Airy disk both microscopes have the same theoretical transverse resolution, although in practice the confocal microscope is preferred because of its axial sectioning. The MTF curves in Fig. 3 also show that the use of an annular illumination pupil enhances the higher spatial frequency content slightly while maintaining or attenuating the intermediate and lower spatial frequencies. It is worth noting that the range of frequencies that are attenuated is between 0 and $f_{\text {max }}(1+\varepsilon) / 2$, thus indicating that the value of $\varepsilon$ used should be chosen with the spatial frequency content of the object of interest in mind.

The use of annular imaging pupils reduces the MTF amplitude across its whole bandwidth but more so for the higher spatial frequency range, when compared to the MTF of the unapodized system. This is consistent with the fact that no transverse resolution improvement is predicted for this apodization configuration (see second row, leftmost plot in Fig. 2).

The changes in the MTF of the simultaneously apodized illumination and imaging pupils can be coarsely described as those introduced by the annular illumination attenuated by the annular imaging pupil.

Images of the photoreceptor mosaic were collected in two subjects with no known history of eye disease. Written informed consent was obtained after the nature and possible risks of the experiment were explained to the subjects. The study was approved by the Institutional Review Boards at the University of Rochester and the Medical College of Wisconsin. The pupil of the imaged eye was dilated and cycloplegia was induced by using one drop of a combination of phenylephrine hydrochloride (2.5\%) and tropicamide (1\%), applied topically. The subjects were aligned and stabilized in front of the AOSLO using a dental impression on a bite bar. Photoreceptor images were collected at the fovea and at a rod dominated region approximately $10^{\circ}$ temporal to the foveal center (determined by fixation).

The two identical AOSLOs used in this study were described previously [10]. The only modification was the placement of circular opaque masks centered in pupil conjugate planes of the illumination (immediate to the light source) and/or imaging paths (immediate to detector). The illumination and imaging paths of the wavefront sensor were not apodized, using full circular pupils both in the illumination and imaging paths. The mask's central obscuration was held by a single, 0.5 mm thick post. Masks with relative radii $\varepsilon=0.26,0.39$ and 0.52 were used. Whenever the illumination and imaging pupils were apodized simultaneously, equal sized masks were used. Images for the different apodization configurations were collected within minutes of each other for a given $\varepsilon$, in random order.

The light collected through a 1.0 Airy disk diameter confocal aperture was detected by a photomultiplier tube (PMT) with gain adjusted to maintain an approximately constant mean pixel value across apodization configurations. An additional set of image sequences were collected at a fixed PMT gain to analyze the reduction of photoreceptor reflectance signal due to the SCE. A superluminescent diode with a peak wavelength of $680 \mathrm{~nm}$ and $8.5 \mathrm{~nm}$ full-width at half-maximum bandwidth (46 $\mu \mathrm{m}$ estimated coherence length) was used as the imaging light source. This source delivered a fixed power to the illumination pupil that in the absence of an obscuration mask was $150 \mu \mathrm{W}$ at the eye over a $7.75 \mathrm{~mm}$ diameter pupil. The images were recorded over a $1.0^{\circ}$ square field of view. At every retinal location and for each $\varepsilon$, sequences of 200 images were recorded at approximately 17 frames per second for each of the four possible pupil configurations. This amounted to a maximum retinal radiant exposure five times below the ANSI maximum permissible exposure [37]. Fifty images from each sequence were registered using the method outlined in [38] and then averaged to increase SNR.

\section{Results and discussion}

Go to:

\subsection{Foveal photoreceptor mosaic}

Registered averages of image sequences captured at $0.5^{\circ}$ from the foveal center are shown in Fig. 4 where some minor resolution improvement due to the use of apodizing masks in the illumination path can be observed for $\varepsilon=0.26$ and 0.39 . Careful 
examination of the image corresponding to $\varepsilon=0.52$ and annular pupil illumination (rightmost image on the second row) reveals that the intensity profiles of the cone photoreceptors appear multi-modal. These multi-modal patterns were first observed ex vivo by Enoch [39] and in vivo in humans by Dubra et al. [25] and are thought to be due to the optical wave-guiding properties of photoreceptors.

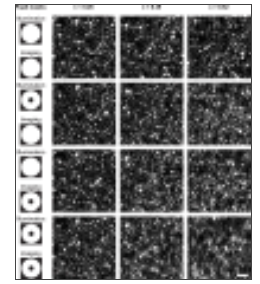

\section{Fig. 4}

Registered averages of foveal cone photoreceptor AOSLO images. Each column corresponds to a different apodization mask normalized radius $\varepsilon$, while each row corresponds to a different apodization configuration. For each $\varepsilon$ all images show ...

Attempts to quantify changes in transverse resolution or image contrast using global sharpness metrics, such as those proposed by Muller and Buffington [40], proved inconclusive due to the temporal fluctuations of the photoreceptor reflectance [11, 41,42$]$. A coarse estimation of the size of photoreceptors can be obtained by measuring the width of the central lobe of the image normalized autocorrelation, using the normalized correlation of the image with itself as defined in Ref. [38]. The change in full-width half-maximum (FWHM) values of the central lobe of the autocorrelation as a result of using annular pupils for all the images in Fig. 4 are shown in Table 1 below. These numbers loosely agree with the theoretical predictions that apodization of the illumination pupil translates to a sharpening of the cone photoreceptor intensity profiles and apodization of the imaging pupil does not. The low signal in the raw images that result from apodizing both pupils degrade the quality of the registration, resulting in blurrier images, as is most noticeable for $\varepsilon=0.39$ and 0.52 .

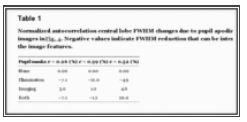

Table 1

Normalized autocorrelation central lobe FWHM changes due to pupil apodization corresponding to images in Fig. 4. Negative values indicate FWHM reduction that can be interpreted as sharpening of the image features.

As predicted by the MTF calculations, the image radial spectra and their ratios (shown in Fig. 5 ) show an enhancement of the higher spatial frequencies below the cutoff frequency $f_{\max }$ relative to the lower spatial frequencies. This enhancement is negligible for the apodization of the imaging pupil and maximal for the apodization of the illumination pupil, as predicted by the theoretical calculations. Interestingly, because the range of (low) frequencies that are attenuated increases with $\varepsilon$, choosing a mask with too large a central obscuration can have the detrimental effect of attenuating the spatial frequencies that correspond to the object of interest (in this case, the photoreceptors), as it appears to be the case for $\varepsilon=0.52$.

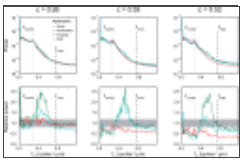

Fig. 5

The top row shows the radial spectra of the foveal cone photoreceptor images in Fig. 4 , with each column corresponding to a different apodizing mask inner radius $\varepsilon$, normalized to unit amplitude at zero frequency. The second row shows the ratios ...

As expected, obscuring the center of either pupil affects the amount of light reaching the detector. To quantify this change and compare it to the values predicted by the SCE, the imaging was repeated with constant PMT gain. Despite the photoreceptor reflectance fluctuations $[\underline{11}, \underline{41}, \underline{42}]$ and other sources of variability such as image registration, tear film and subject alignment, the observed reflectance amplitude values (shown in Table 2 ) are consistent with the SCE predictions.

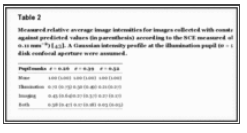

Table 2

Measured relative average image intensities for images collected with constant

PMT gain compared against predicted values (in parenthesis) according to the SCE measured objectively at fixation $\left(\rho=0.11 \mathrm{~mm}^{-2}\right)[\underline{43}]$. A Gaussian intensity ...

The practical use of the proposed apodization for in vivo imaging of the photoreceptor mosaic should ultimately be assessed based on whether it improves our ability to identify photoreceptors. To this effect, we performed cell counts using a semi-automated algorithm developed by Li and Roorda [44] and further automated by Garrioch et al. [45]. Manual counting was also performed for comparison, twice on every image by three trained observers. The results from both counting methods, summarized in Table 3 , are in agreement with the previous observations. The use of apodization masks with $\varepsilon=0.26$ and 0.39 in the illumination path allows the identification of 8 to $20 \%$ more cone photoreceptors, with both the automated and manual counting. The multi-modal 
appearance of the cones for the image corresponding to an annular illumination pupil with $\varepsilon=0.52$ led to a large number of false counts, highlighting the fact that the inner radius of the apodizing mask must be selected with some care. The apodization of the imaging pupil with the two smaller masks yields comparable cone counts to those resulting from the unapodized configuration, as expected from the theoretical predictions.

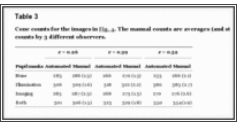

Table 3

Cone counts for the images in Fig. 4. The manual counts are averages (and standard deviation) from 6 counts by 3 different observers.

Interestingly, the multi-modal intensity patterns of the cone photoreceptors are either not as dramatic or not observed at all when using a circular illumination pupil and an annular imaging pupil. This is probably not due to an asymmetry in the SCE, but to the blur induced by the convolution of the imaging PSF and the confocal pinhole transmission in Eq. (3).

\subsection{Photoreceptor mosaic at $10^{\circ}$ temporal}

At $10^{\circ}$ temporal from fixation (Fig. 6 ) the rod photoreceptors are much smaller than the cones. As with the foveal images, more photoreceptors can be identified when the illumination pupil is apodized. The radially averaged power spectra of these retinal images and their ratios, shown in Fig. 7 , closely resemble those in Fig. 5 and are consistent with the theoretical calculations.

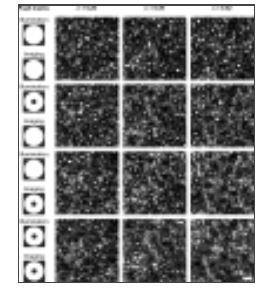

\section{Fig. 6}

Registered averages of photoreceptor AOSLO images at $10^{\circ}$ temporal from fixation. Each column corresponds to a different apodization mask normalized radius $\varepsilon$, while each row corresponds to a different apodization configuration. For each ...

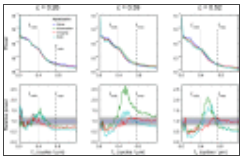

Fig. 7

Radial spectra of the rod-dominated images in Fig. 6, with each column representing a different $\varepsilon$. The second row shows ratios of the normalized spectra from images taken with annular pupil(s) to the spectra of images taken with circular pupils. ...

The changes in autocorrelation central lobe FWHM as a result of the pupil apodization (shown in Table 4 ) are consistent with those observed when imaging the foveal cones. Interestingly, the largest change occurs when using the $\varepsilon=0.39$ mask which was also the case for foveal cones, suggesting that this might be the best of the three masks (and configuration) for photoreceptor imaging.

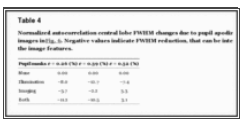

$\underline{\text { Table } 4}$

Normalized autocorrelation central lobe FWHM changes due to pupil apodization corresponding to images in Fig. 6. Negative values indicate FWHM reduction, that can be interpreted as sharpening of the image features.

Previous SCE studies show both through objective and subjective measurements that peripheral cone photoreceptors are more directionally sensitive than cones near the center of the fovea $[\underline{39}, \underline{46}]$, and that the directional sensitivity of rod photoreceptors is similar to that of foveal cones $[\underline{47}, \underline{48}]$. By repeating the imaging shown in Fig. 6 with constant PMT gain, we recorded the mean relative photoreceptor intensity values presented in Table 5 below, that suggest an SCE described by lower $\rho$ values than those published in the literature. This could be explained by the fact that the AOSLO images are focused on the reflections from the inner-outer segment interface, which according to the work by Gao et al. [49] are less directional than those from the posterior surface of the photoreceptor outer segments. As a consequence of the difference in SCE between rods and cones, the ratio of cone to rod signal decreases as $\varepsilon$ increases (see Table 6 ). This relative intensity change could be exploited to automate the identification of cones and rods as follows. Two images one with and one without apodization can be collected and once each cell has been counted, the decision of which cell is a cone or a rod can be made based on the relative change of brightness between the two images.

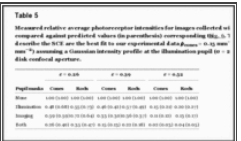

Table 5

Measured relative average photoreceptor intensities for images collected with constant PMT gain compared against predicted values (in parenthesis) 
corresponding to Fig. 6. The values used to describe the SCE are the best fit to our experimental data $(\ldots$

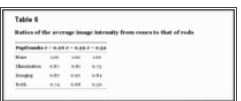

Table 6

Ratios of the average image intensity from cones to that of rods

Lastly, manual cone counts, followed by automated and manual rod counts were performed on the images in Fig. 6 . Once the cones were manually counted, the automated software counted all the bright spots as individual photoreceptors. Then the counts within 6 pixels of the manually entered cone locations were removed as misidentified rods. The remaining identified bright spots were counted as rods. The results, summarized in Table 7 further support the previous observations, showing the highest rod count for the imaging configurations with apodized illumination only, followed by the case with both illumination and imaging apodized. No consistent benefit or degradation was observed from apodizing only the imaging pupil. As with the cone images, the increase in photoreceptor counts is non-negligible (between 10 and 29\%) when using annular illumination pupils, demonstrating the practical utility of the method.

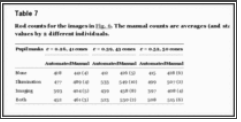

Table 7

Rod counts for the images in Fig. 6. The manual counts are averages (and standard deviation) from 4 values by 2 different individuals.

The use of centered, annular pupil illumination and/or imaging pupils was evaluated in a reflectance AOSLO as a method for increasing transverse resolution with low cost and complexity. Images from the photoreceptor mosaic at retinal eccentricities with very different spatial frequency content were collected and analyzed. Despite not considering the wave-guiding or scattering properties of the photoreceptors in the theoretical calculation, the experimental findings are in agreement with the predictions from paraxial confocal microscopy theory. This indicates that the use of annular pupil illumination and full circular imaging pupil provide highest transverse resolution. The two annular illumination pupils with smaller inner radii performed best, whether measured in terms of the narrowing of the autocorrelation central lobe or the manual and automated photoreceptor counts. The use of the annular apodizing mask with the largest inner radius $(\varepsilon=0.52)$ provided different outcomes depending on the size of the photoreceptors; when imaging rods, the use of the mask improved their visibility as measured with the autocorrelation and counts, while when imaging cones their intensity profile could become multi-modal. These multi-modal intensity profiles could lead to incorrect high cell counts as each lobe within the pattern might be identified as a rod or another cone. Therefore, the size of the apodizing mask must be chosen with some knowledge of the spatial frequency content and optical properties of the structure to be imaged.

The appearance or enhancement of multi-modal patterns when illuminating the pupil with a non-uniform intensity profile is consistent with the wave-guiding properties of photoreceptors and it could be exploited to gain insight into the structure of photoreceptors and their dynamics. For example, by placing a spatial light modulator in the pupil plane and collecting images with different intensity patterns (not just annular, as demonstrated in this work), information on the modes of each photoreceptor could be obtained and used in combination with wave-guiding theory to infer the geometry of each photoreceptor. Alternatively, a fixed illumination pattern (e.g. annular) could be used to monitor the evolution of the multi-modal patterns, to determine if subtle transverse changes take place in the photoreceptor mosaic. This could be used to study the disc shedding and renewal process, in a similar way that the evolution of the fundamental modes has been used before $[\underline{11}, \underline{42}, \underline{50}-\underline{52}]$. If the multi-modal intensity profiles are not affected by daily changes, they could potentially reveal early changes in photoreceptor disease such as swelling.

We found that the use of apodizing masks affected the average photoreceptor intensity as predicted by the SCE measurements reported in the literature. The fact that the SCE is different for cones and rods at any given retinal location means that cone intensity can be differentially attenuated with respect to that of rods through pupil apodization. By capturing pairs of images of the same retinal location with and without pupil apodization, the cone photoreceptors could be differentiated from rods. One potential imaging protocol is as follows: first, capture an image sequence with annular pupil illumination and $\varepsilon$ between 0.3 and 0.4 for best transverse resolution. Next capture a pair of image sequences with and without apodization in both illumination and imaging pupils. A difference map, created from the pair of sequences can be used to identify the photoreceptors in the first image sequence.

A consequence of the use of the apodization masks evaluated in this work is an axial elongation of the PSF. Although this fact can be seen as detrimental in confocal scanning ophthalmoscopy, it could be beneficial for AOOCT. The axial resolution of most current OCT ophthalmoscopes is proportional to the bandwidth of the source and mostly independent of the axial PSF, with current instruments boasting axial resolution of up to $3 \mu \mathrm{m}$. When imaging the retina using AOOCT with a full pupil, the lateral resolution 
degrades axially outside the PSF [13]. The use of annular illumination would extend the PSF axially and thus increase the range of retinal layers that can be imaged simultaneously without loss of transverse and axial resolution. In fact, the former would be improved.

Finally, undesired back-reflections from the surfaces of refractive elements in most current AO ophthalmoscopes are removed by slightly tilting the elements. Placing an obscuration in the illumination path as demonstrated here could be used to mitigate these back-reflections without having to tilt these elements [53], thus potentially improving the optical performance of refractive AO ophthalmoscopes. The on-axis nature of refractive systems, coupled with the additional surfaces for aberration control might allow for smaller instruments.

Alfredo Dubra-Suarez, Ph.D., holds a Career Award at the Scientific Interface from the Burroughs Welcome Fund. This research was supported financially by Research to Prevent Blindness. The authors would like to thank Drew Scoles, Jennifer Norris and Melissa Diederichs for their assistance with experiments and data processing, and Ben Masella and Melissa Geng for manuscript revisions.

1. Liang J., Williams D. R., Miller D. T., "Supernormal vision and high-resolution retinal imaging through adaptive optics," J. Opt. Soc. Am. A 14(11), 2884-2892 (1997).10.1364/JOSAA.14.002884 [PubMed] [Cross Ref]

2. Choi S. S., Doble N., Hardy J. L., Jones S. M., Keltner J. L., Olivier S. S., Werner J. S., “In vivo imaging of the photoreceptor mosaic in retinal dystrophies and correlations with visual function," Invest. Ophthalmol. Vis. Sci. 47(5), 2080-2092

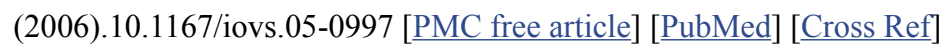

3. Rha J., Jonnal R. S., Thorn K. E., Qu J., Zhang Y., Miller D. T., “Adaptive optics flood-illumination camera for high speed retinal imaging," Opt. Express 14(10), 4552-4569 (2006).10.1364/OE.14.004552 [릴ed] [Cross Ref]

4. Rha J., Schroeder B., Godara P., Carroll J., "Variable optical activation of human cone photoreceptors visualized using a short coherence light source," Opt. Lett. 34(24), 3782-3784 (2009).10.1364/OL.34.003782 [PMC free article] [PubMed] [ㄷoss Ref]

5. Dees E. W., Dubra A., Baraas R. C., "Variability in parafoveal cone mosaic in normal trichromatic individuals,” Biomed. Opt. Express 2(5), 1351-1358 (2011).10.1364/BOE.2.001351 [PMC free article] [PubMed] [Cross Ref]

6. Roorda A., Romero-Borja F., Donnelly Iii W., Queener H., Hebert T. J., Campbell M. C. W., “Adaptive optics scanning laser ophthalmoscopy," Opt. Express 10(9), 405-412 (2002). [PubMed]

7. Hammer D. X., Ferguson R. D., Bigelow C. E., Iftimia N. V., Ustun T. E., Burns S. A., “Adaptive optics scanning laser ophthalmoscope for stabilized retinal imaging," Opt. Express 14(8), 3354-3367 (2006).10.1364/OE.14.003354 [PMC free article] [PubMed] [Cross Ref]

8. Gray D. C., Merigan W., Wolfing J. I., Gee B. P., Porter J., Dubra A., Twietmeyer T. H., Ahamd K., Tumbar R., Reinholz F., Williams D. R., "In vivo fluorescence imaging of primate retinal ganglion cells and retinal pigment epithelial cells," Opt. Express 14(16), 7144-7158 (2006).10.1364/OE.14.007144 [릴ed] [Cross Ref]

9. Vohnsen B., Rativa D., “Ultrasmall spot size scanning laser ophthalmoscopy,” Biomed. Opt. Express 2(6), 1597-1609 (2011).10.1364/BOE.2.001597 [PMC free article] [PubMed] [Cross Ref]

10. Dubra A., Sulai Y., "Reflective afocal broadband adaptive optics scanning ophthalmoscope,” Biomed. Opt. Express 2(6), 1757-1768 (2011).10.1364/BOE.2.001757 [PMC free article] [PubMed] [Cross Ref]

11. Cooper R. F., Dubis A. M., Pavaskar A., Rha J., Dubra A., Carroll J., "Spatial and temporal variation of rod photoreceptor reflectance in the human retina," Biomed. Opt. Express 2(9), 2577-2589 (2011).10.1364/BOE.2.002577 [PMC free article] [PubMed] [Cross Ref]

12. Hermann B., Fernández E. J., Unterhuber A., Sattmann H., Fercher A. F., Drexler W., Prieto P. M., Artal P., “Adaptive-optics ultrahigh-resolution optical coherence tomography,” Opt. Lett. 29(18), 2142-2144 (2004).10.1364/OL.29.002142 [PubMed] [Cross Ref]

13. Zhang Y., Rha J., Jonnal R., Miller D., “Adaptive optics parallel spectral domain optical coherence tomography for imaging the living retina,” Opt. Express 13(12), 4792-4811 (2005).10.1364/OPEX.13.004792 [PubMed] [Cross Ref]

14. Zawadzki R. J., Jones S. M., Olivier S. S., Zhao M., Bower B. A., Izatt J. A., Choi S., Laut S., Werner J. S., “Adaptive-optics 
optical coherence tomography for high-resolution and high-speed 3D retinal in vivo imaging," Opt. Express 13(21), 8532-8546 (2005).10.1364/OPEX.13.008532 [PMC free article] [PubMed] [Cross Ref]

15. Bigelow C. E., Iftimia N. V., Ferguson R. D., Ustun T. E., Bloom B., Hammer D. X., “Compact multimodal adaptive-optics spectral-domain optical coherence tomography instrument for retinal imaging,” J. Opt. Soc. Am. A 24(5), 1327-1336

(2007).10.1364/JOSAA.24.001327 [PubMed] [Cross Ref]

16. Zhang Y., Cense B., Rha J., Jonnal R. S., Gao W., Zawadzki R. J., Werner J. S., Jones S., Olivier S., Miller D. T., “High-speed volumetric imaging of cone photoreceptors with adaptive optics spectral-domain optical coherence tomography," Opt. Express 14(10), 4380-4394 (2006).10.1364/OE.14.004380 [M free article] [PubMed] [Cross Ref]

17. Roorda A., Zhang Y., Duncan J. L., "High-resolution in vivo imaging of the RPE mosaic in eyes with retinal disease," Invest. Ophthalmol. Vis. Sci. 48(5), 2297-2303 (2007).10.1167/iovs.06-1450 [PubMed] [Cross Ref]

18. Morgan J. I. W., Dubra A., Wolfe R., Merigan W. H., Williams D. R., "In vivo autofluorescence imaging of the human and macaque retinal pigment epithelial cell mosaic,” Invest. Ophthalmol. Vis. Sci. 50(3), 1350-1359 (2009).10.1167/iovs.08-2618 [PMC free article] [PubMed] [Cross Ref]

19. Martin J. A., Roorda A., "Direct and noninvasive assessment of parafoveal capillary leukocyte velocity," Ophthalmology 112(12), 2219-2224 (2005).10.1016/j.ophtha.2005.06.033 [PubMed] [Cross Ref]

20. Cense B., Koperda E., Brown J. M., Kocaoglu O. P., Gao W., Jonnal R. S., Miller D. T., "Volumetric retinal imaging with ultrahigh-resolution spectral-domain optical coherence tomography and adaptive optics using two broadband light sources," Opt.

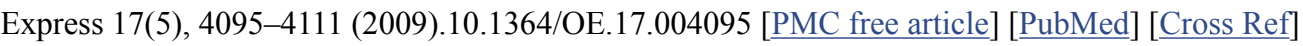

21. Scoles D., Gray D. C., Hunter J. J., Wolfe R., Gee B. P., Geng Y., Masella B. D., Libby R. T., Russell S., Williams D. R., Merigan W. H., "In-vivo imaging of retinal nerve fiber layer vasculature: imaging — histology comparison," BMC Ophthalmol. 9, 9 (2009).10.1186/1471-2415-9-9 [MC free article] [PubMed] [Cross Ref]

22. Vilupuru A. S., Rangaswamy N. V., Frishman L. J., Smith E. L., 3rd, Harwerth R. S., Roorda A., "Adaptive optics scanning laser ophthalmoscopy for in vivo imaging of lamina cribrosa,” J. Opt. Soc. Am. A 24(5), 1417-1425 (2007).10.1364/JOSAA.24.001417 [PubMed] [Cross Ref]

23. Torti C., Povazay B., Hofer B., Unterhuber A., Carroll J., Ahnelt P. K., Drexler W., “Adaptive optics optical coherence tomography at 120,000 depth scans/s for non-invasive cellular phenotyping of the living human retina," Opt. Express 17(22), 19382-19400 (2009).10.1364/OE.17.019382 [PMC free article] [PubMed] [Cross Ref]

24. Ivers K. M., Li C., Patel N., Sredar N., Luo X., Queener H., Harwerth R. S., Porter J., "Reproducibility of measuring lamina cribrosa pore geometry in human and nonhuman primates with in vivo adaptive optics imaging," Invest. Ophthalmol. Vis. Sci. 52(8), 5473-5480 (2011).10.1167/iovs.11-7347 [PMC free article] [PubMed] [Cross Ref]

25. Dubra A., Sulai Y., Norris J. L., Cooper R. F., Dubis A. M., Williams D. R., Carroll J., "Noninvasive imaging of the human rod photoreceptor mosaic using a confocal adaptive optics scanning ophthalmoscope," Biomed. Opt. Express 2(7), 1864-1876 (2011).10.1364/BOE.2.001864 [PMC free article] [PubMed] [Cross Ref]

26. Merino D., Duncan J. L., Tiruveedhula P., Roorda A., "Observation of cone and rod photoreceptors in normal subjects and patients using a new generation adaptive optics scanning laser ophthalmoscope,” Biomed. Opt. Express 2(8), 2189-2201 (2011).10.1364/BOE.2.002189 [PMC free article] [PubMed] [Cross Ref]

27. Birren J. E., Casperson R. C., Botwinick J., “Age changes in pupil size,” J. Gerontol. 5(3), 216-221 (1950).10.1093/geronj 15.3.216 [PubMed] [Cross Ref]

28. Friedman D. S., O’Colmain B. J., Muñoz B., Tomany S. C., McCarty C., de Jong P. T., Nemesure B., Mitchell P., Kempen J., Eye Diseases Prevalence Research Group , "Prevalence of age-related macular degeneration in the United States," Arch. Ophthalmol. 122(4), 564-572 (2004).10.1001/archopht.122.4.564 [PubMed] [Cross Ref]

29. Friedman D. S., Wolfs R. C., O’Colmain B. J., Klein B. E., Taylor H. R., West S., Leske M. C., Mitchell P., Congdon N., Kempen J., Eye Diseases Prevalence Research Group , "Prevalence of open-angle glaucoma among adults in the United States," Arch. Ophthalmol. 122(4), 532-538 (2004).10.1001/archopht.122.4.532 [PMC free article] [PubMed] [Cross Ref]

30. Kempen J. H., O’Colmain B. J., Leske M. C., Haffner S. M., Klein R., Moss S. E., Taylor H. R., Hamman R. F., Eye Diseases Prevalence Research Group, "The prevalence of diabetic retinopathy among adults in the United States,” Arch. Ophthalmol. 122(4), 552-563 (2004).10.1001/archopht.122.4.552 [PubMed] [Cross Ref] 
31. Tschunko H. F. A., “Imaging performance of annular apertures,” Appl. Opt. 13(8), 1820-1823 (1974).10.1364/AO.13.001820 [PubMed] [Cross Ref]

32. Wilson T., Hewlett S. J., "The use of annular pupil plane filters to tune the imaging properties in confocal microscopy," J. Mod. Opt. 37(12), 2025-2046 (1990).10.1080/09500349014552221 [Cross Ref]

33. Rayleigh L., "Investigations in optics with special reference to the spectroscope," Philos. Mag. 5(8), 261-274 (1879).

34. Gustafsson M. G. L., "Surpassing the lateral resolution limit by a factor of two using structured illumination microscopy," J. Microsc. 198(2), 82-87 (2000).10.1046/j.1365-2818.2000.00710.x [PubMed] [Cross Ref]

35. M. Born and E. Wolf, "Principles of optics." 7th (expanded) Edition (Cambridge University press) p. 487 (1999).

36. Wilson T., Carlini A. R., "Size of the detector in confocal imaging systems," Opt. Lett. 12(4), 227-229

(1987).10.1364/OL.12.000227 [PubMed] [Cross Ref]

37. Delori F. C., Webb R. H., Sliney D. H., American National Standards Institute , "Maximum permissible exposures for ocular safety (ANSI 2000), with emphasis on ophthalmic devices,” J. Opt. Soc. Am. A 24(5), 1250-1265

(2007).10.1364/JOSAA.24.001250 [PubMed] [Cross Ref]

38. Dubra A., Harvey Z., "Registration of 2d images from fast scanning ophthalmic instruments,” Lect. Notes Comput. Sci. 6204, 60-71 (2010).10.1007/978-3-642-14366-3_6 [Cross Ref]

39. Enoch J. M., “Optical properties of the retinal receptors,” J. Opt. Soc. Am. 53(1), 71-85 (1963).10.1364/JOSA.53.000071

[Cross Ref]

40. Muller R. A., Buffington A., "Real-time correction of atmospherically degraded telescope images through image sharpening," J. Opt. Soc. Am. 64(9), 1200-1210 (1974).10.1364/JOSA.64.001200 [Cross Ref]

41. Pallikaris A., Williams D. R., Hofer H., “The reflectance of single cones in the living human eye,” Invest. Ophthalmol. Vis. Sci. 44(10), 4580-4592 (2003).10.1167/iovs.03-0094 [PubMed] [Cross Ref]

42. Jonnal R. S., Besecker J. R., Derby J. C., Kocaoglu O. P., Cense B., Gao W., Wang Q., Miller D. T., "Imaging outer segment renewal in living human cone photoreceptors," Opt. Express 18(5), 5257-5270 (2010).10.1364/OE.18.005257 [PMC free article] [PubMed] [Cross Ref]

43. Burns S. A., Wu S., He J. C., Elsner A. E., "Variations in photoreceptor directionality across the central retina," J. Opt. Soc. Am. A 14(9), 2033-2040 (1997).10.1364/JOSAA.14.002033 [PMC free article] [PubMed] [Cross Ref]

44. Li K. Y., Roorda A., “Automated identification of cone photoreceptors in adaptive optics retinal images,” J. Opt. Soc. Am. A 24(5), 1358-1363 (2007).10.1364/JOSAA.24.001358 [PubMed] [Cross Ref]

45. Garrioch R., Langlo C., Dubis A. M., Cooper R. F., Dubra A., Carroll J., "Repeatability of in vivo parafoveal cone density and spacing measurements,” Optom. Vis. Sci. 89(5), 632-643 (2012).10.1097/OPX.0b013e3182540562 [PMC free article] [PubMed] [Cross Ref]

46. Burns S. A., Wu S., Delori F., Elsner A. E., "Direct measurement of human-cone-photoreceptor alignment," J. Opt. Soc. Am. A 12(10), 2329-2338 (1995).10.1364/JOSAA.12.002329 [PubMed] [Cross Ref]

47. Van Loo J. A., Jr, Enoch J. M., “The scotopic Stiles-Crawford effect,” Vision Res. 15(8-9), 1005-1009 (1975).10.1016/0042-6989(75)90243-6 [PubMed] [Cross Ref]

48. Alpern M., Ching C. C., Kitahara K., “The directional sensitivity of retinal rods,” J. Physiol. 343, 577-592 (1983). [PMC free article] [PubMed]

49. Gao W., Cense B., Zhang Y., Jonnal R. S., Miller D. T., "Measuring retinal contributions to the optical Stiles-Crawford effect with optical coherence tomography," Opt. Express 16(9), 6486-6501 (2008).10.1364/OE.16.006486 [PMC free article] [PubMed] [Cross Ref]

50. Jonnal R. S., Rha J., Zhang Y., Cense B., Gao W., Miller D. T., "In vivo functional imaging of human cone photoreceptors,” Opt. Express 15(24), 16141-16160 (2007).10.1364/OE.15.016141 [MC free article] [PubMed] [Cross Ref]

51. Kocaoglu O. P., Lee S., Jonnal R. S., Wang Q., Herde A. E., Derby J. C., Gao W., Miller D. T., "Imaging cone photoreceptors in three dimensions and in time using ultrahigh resolution optical coherence tomography with adaptive optics," Biomed. Opt.

Express 2(4), 748-763 (2011).10.1364/BOE.2.000748 [Mㅡ free article] [PubMed] [Cross Ref] 
52. Pircher M., Kroisamer J. S., Felberer F., Sattmann H., Götzinger E., Hitzenberger C. K., “Temporal changes of human cone photoreceptors observed in vivo with SLO/OCT," Biomed. Opt. Express 2(1), 100-112 (2011).10.1364/BOE.2.000100 [PMC free article] [PubMed] [Cross Ref]

53. Geng Y., Dubra A., Yin L., Merigan W. H., Sharma R., Libby R. T., Williams D. R., “Adaptive optics retinal imaging in the living mouse eye," Biomed. Opt. Express 3(4), 715-734 (2012).10.1364/BOE.3.000715 [PMC free article] [PubMed] [Cross Ref]

Articles from Biomedical Optics Express are provided here courtesy of Optical Society of America 